\title{
Development of an Abort Gap Monitor for High-Energy Proton Rings
}

\author{
J.-F. Beche, J. Byrd, S. De Santis, P. Denes, M. Placidi, W. Turner, \\ M. Zolotorev
}

Lawrence Berkeley National Laboratory, Berkeley, USA

\begin{abstract}
The fill pattern in proton synchrotrons usually features an empty gap, longer than the abort kicker raise time, for machine protection. This gap is referred to as the "abort gap" and any particles, which may accumulate in it due to injection errors and diffusion between RF buckets, would be lost inside the ring, rather than in the beam dump, during the kicker firing. In large proton rings, due to the high energies involved, it is vital to monitor the build up of charges in the abort gap with a high sensitivity. We present a study of an abort gap monitor based on a photomultiplier with a gated microchannel plate, which would allow for detecting low charge densities by monitoring the synchrotron radiation emitted. We show results of beam test experiments at the Advanced Light Source using a Hamamatsu 5916U MCP-PMT and compare them to the specifications for the Large Hadron Collider
\end{abstract}

\section{INTRODUCTION}

Large proton synchrotron rings are characterized by very high energies stored in their beams and extensive use of superconducting magnets. Such magnets, as well as other delicate components, can easily be damaged by even a very small fraction of the beam, if that should be hitting them. Special beam dumps, capable to absorb such high stored energies $(\sim 350 \mathrm{MJ} /$ beam in the LHC, for example) are placed along the ring together with abort kicker magnets which can steer the entire beam to be lost in the dump in case of irregular operations. It is standard practice to leave a gap, longer than the abort kicker raise time, in the ring fill pattern to avoid dispersing particles on other machine components during the kicker firing. It is vital to assure that no particles accumulate in the gap since those would receive only a kick of reduced strength, when the dump system is activated, and would hit machine components. The maximum charge density admissible in the gap can be calculated taking into account the machine energy and the damage threshold of the most sensitive components (usually quenching in superconducting magnets sets the lower limit). The abort gap needs then to be monitored continuously in order to dump the beam or activate gap clearing procedures when the safety threshold is reached.

* This work was supported by the U.S. Department of Energy under Contract No. DE-AC03-76SF00098. 
Several phenomena can cause the gap to become populated: timing errors at injection; drifting of unbunched particles; diffusion of particles between RF buckets; debunching due to glitches in the RF. The time scale of such phenomena is varied, going from practically instantaneous for injection errors to tens of thousands of turns for slow diffusion mechanisms.

In this paper we present a preliminary study of a simple instrument, based on a gated photomultiplier, which is suitable to be included in a ring's interlock chain. Experimental tests have been carried out at the Advanced Light Source and their results are reported here. Though our instrument is not designed with a particular machine in mind, we will often make reference to the Large Hadron Collider for which an exhaustive study of the abort gap monitoring requirements is available [1]. The LHC also makes for an interesting case study due to the necessity of monitoring the abort gap at two different energies $(450 \mathrm{GeV}$ at injection and $7 \mathrm{TeV}$ at final energy) with the relative consequences on the photon flux available and different safety thresholds.

\section{ABORT GAP MONITOR OUTLINE}

The fundamental component of our instrument is a gateable microchannel plate photomultiplier tube (MCP-PMT) which we use for measuring the synchrotron radiation emitted by the circulating particles in a suitable diagnostic station (dipole or undulator). By selecting an appropriately long observation window and shifting it along the abort gap, it is possible to monitor the presence of particles continuously and to estimate their longitudinal density. Commercially available MCP-PMTs [2] are the ideal instrument for such a task due to their high gain, fast gate, low noise and variety of peak wavelength of their spectral response. Also the low voltage required for gating and their reduced dimensions (Fig.1) are other beneficial features that make the use of such a device particularly simple.

The optimal time window used has to be determined on the basis of the particular configuration of the dumping kicker and dump line. Other aspects to take into account are the MCP-PMT maximum duty factor, typically of the order of a few percent, the abort gap length, compared to the total length of the ring, and the maximum allowable integration time, compared to the expected photon flux. As an example, the requirements for the LHC specify maximum number of protons of $4 \cdot 10^{9}(450 \mathrm{GeV})$ and $6 \cdot 10^{6}(7 \mathrm{Tev})$ over $100 \mathrm{~ns}$ intervals. With a $1 \%$ duty factor, it is possible to take $100 \mathrm{~ns}$ long samples only at $10 \mu \mathrm{s}$ intervals, which means one sample per turn given that the abort gap is $3 \mu \mathrm{s}$ and the revolution period $\sim 89 \mu \mathrm{s}$. Mapping the entire abort gap requires 30 of such samples and, therefore, 30 turns. CERN specifications allow for a $100 \mathrm{~ms}$ integration time, which means that it is possible to average over 37 distinct measurements for every $100 \mathrm{~ns}$ sample. This has to be compared to the expected [3] intensity in the longitudinal diagnostic station which is $\sim 40$ photons $/ 0.1 \%$ bandwidth/turn $(450 \mathrm{GeV})$ and $\sim 5$ photons $/ 0.1 \%$ bandwidth/turn $(7 \mathrm{TeV})$ produced by the maximum allowable number of protons at an $800 \mathrm{~nm}$ wavelength. The typical quantum efficiency of an MCP-PMT is in the range of a few percents, but they have a 
fairly large bandwidth so that it is possible to integrate photon counts over a $10 \%$ range which gives a very good signal to noise ratio.

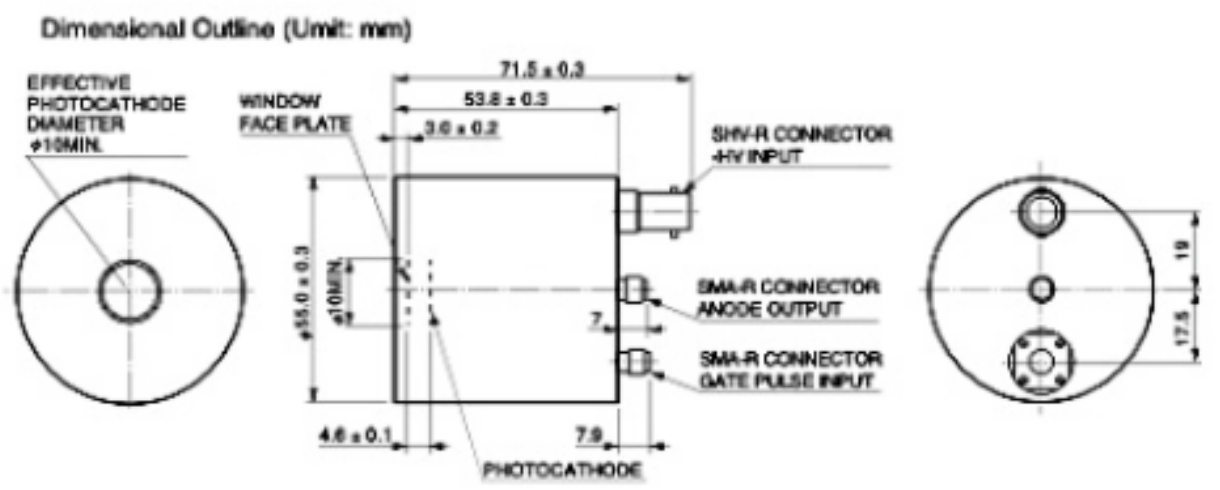

FIGURE 1. Hamamatsu gateable microchannel plate photomultiplier R5916U-50 Series.

Monitoring the beginning and the end of the abort gap presents an extra challenge due to the presence of regular bunches, having a current higher by many orders of magnitude, right next to these regions. The 1 ns gate raise time in our Hamamatsu MCP-PMT is fast enough to ensure that the filled bunches can be gated out.

InsIrument Regeponse Function (IFF)

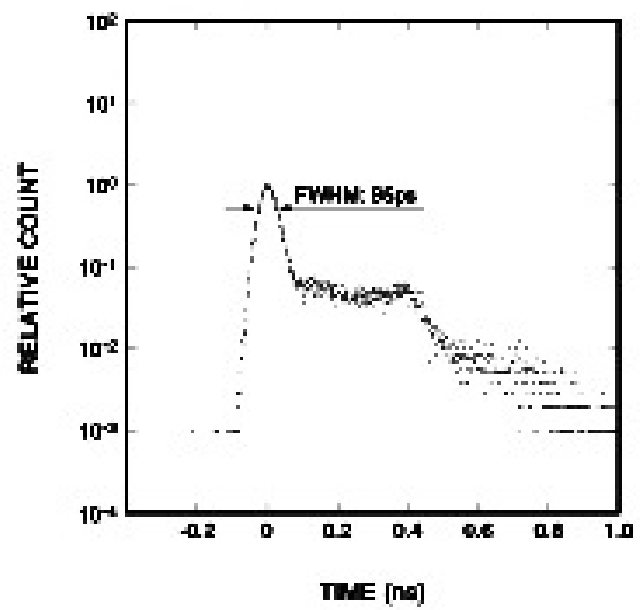

FIGURE 2. Instrument response function or the Hamamatsu R5916U-50 MCP-PMT.

On the other hand, the instrument response function (roughly equivalent to the impulse response) of the MCP-PMT decays three order of magnitude in about $1 \mathrm{~ns}$, so that the question arises on whether the large filled bunches could saturate our instrument, keeping in mind that the separation between RF buckets is only a few nanoseconds in many rings ( $2.5 \mathrm{~ns}$ in the LHC and $19 \mathrm{~ns}$ in the Tevatron, for example). We also wanted to verify that the intense radiation from the filled bunches wouldn't saturate 
the photocatode, even with the gate signal off. In that case a fast optical (Pockels cell) switch in front of the instrument would have been required, adding to the instrument's complexity. To this end we conducted experimental tests at the Advanced Light Source, which features a $\sim 100$ ns long gap in its fill and a bunch separation of 2 ns. Further tests on the instrument's response to unbunched beam will be shortly carried out at the Tevatron.

\section{EXPERIMENTAL RESULTS}

Some parameters of the ALS storage ring make it a good place for testing a possible utilization of a MCP-PMT as an abort gap monitor for the LHC. We mentioned the similar distance between RF buckets and a gap length equal to the length of an LHC sample in the previous paragraph. The ALS ring also features a special high current bunch (referred to as camshaft) in the middle of its fill gap which, after a while, is followed by very low current parasitic bunches due to diffusion of electrons from the camshaft into the following RF buckets. This is particularly interesting from the point of view of studying eventual saturation phenomena in the MCP-PMT, which could not allow to detect a low charge density next to a much larger one.

Our experimental station is at the ALS diagnostic beamline (BL 3.1) which uses the synchrotron radiation generated in a bending dipole $(1.3 \mathrm{~T})$.

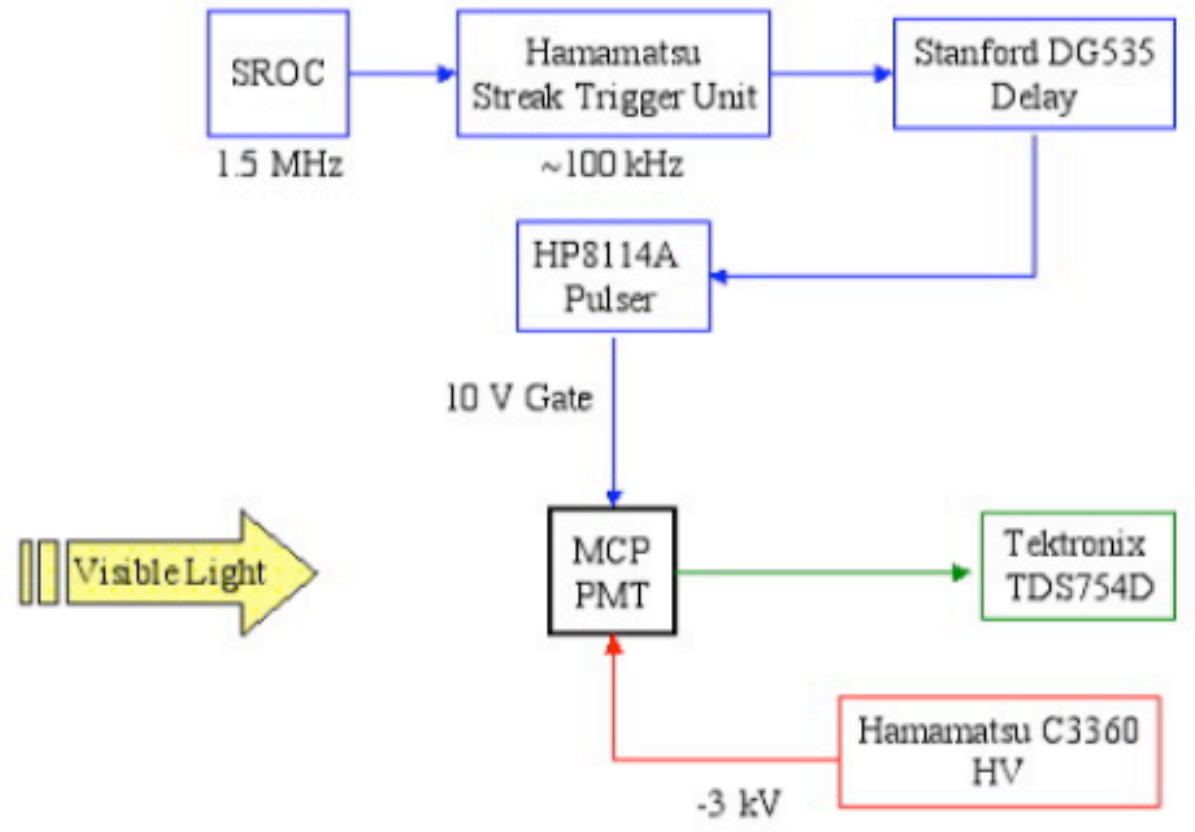

FIGURE 3. Block diagram of the experimental setup. 
Figure 3 shows the block diagram of our experimental setup at BL 3.1. The $10 \mathrm{~V}$ gating pulse, synchronized to the ring orbit clock (SROC), can be shifted around using a delay box and the internal pulser capabilities.

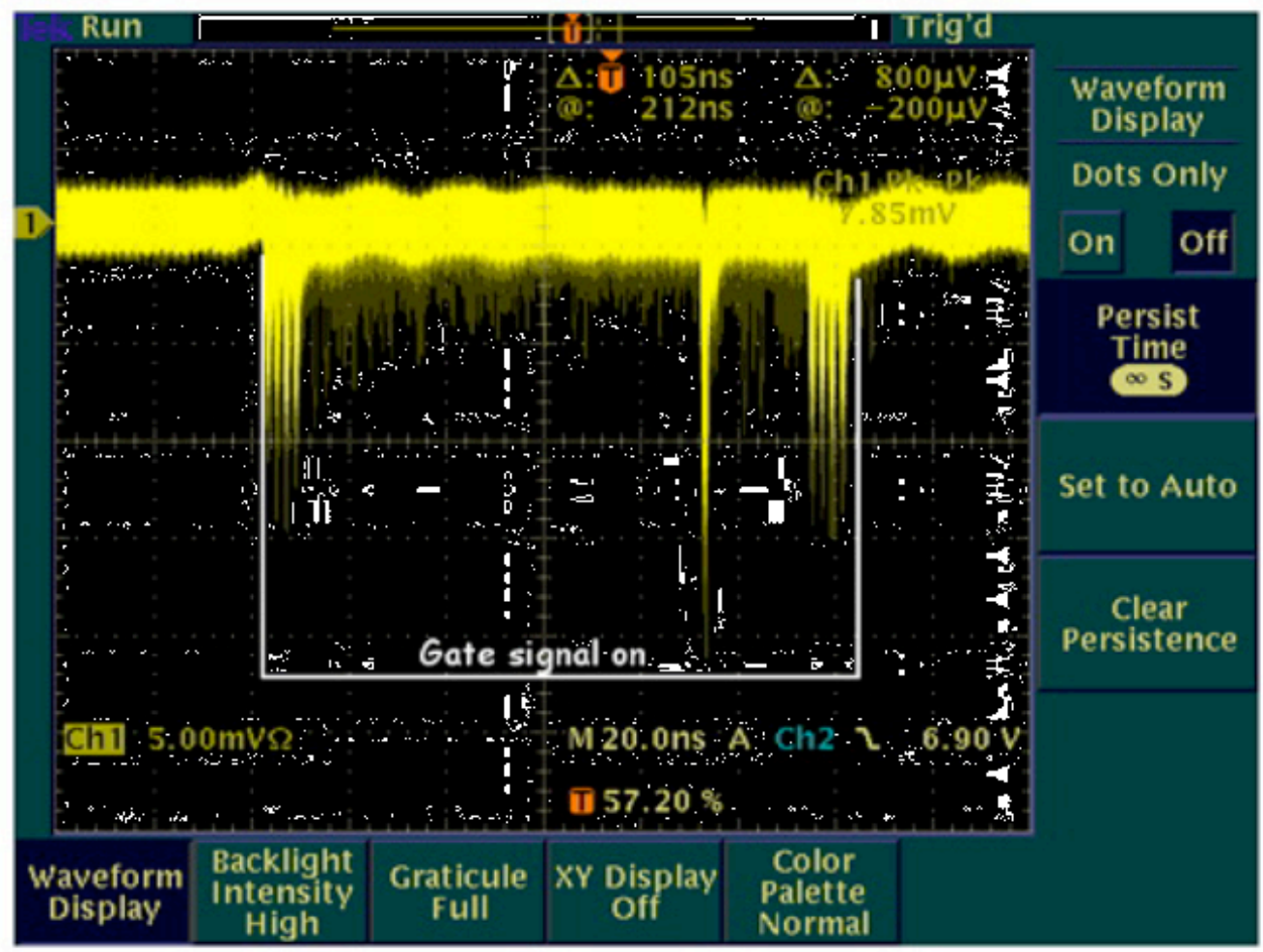

FIGURE 4. The ALS fill gap with camshaft as measured by an MCP-PMT.

Figure 4 shows an image of the entire ALS fill gap. Four regularly filled bunches can be seen at the beginning and at the end of the gap and the camshaft is the larger signal between them. This picture was obtained setting the oscilloscope on an infinite persistence time, so that we are indeed observing data accumulated over a very large number of turns. This was done because, at the beginning of the experiment, we had placed a neutral density filter in front of the MCP-PMT, to be sure not to expose it to excessive light. 


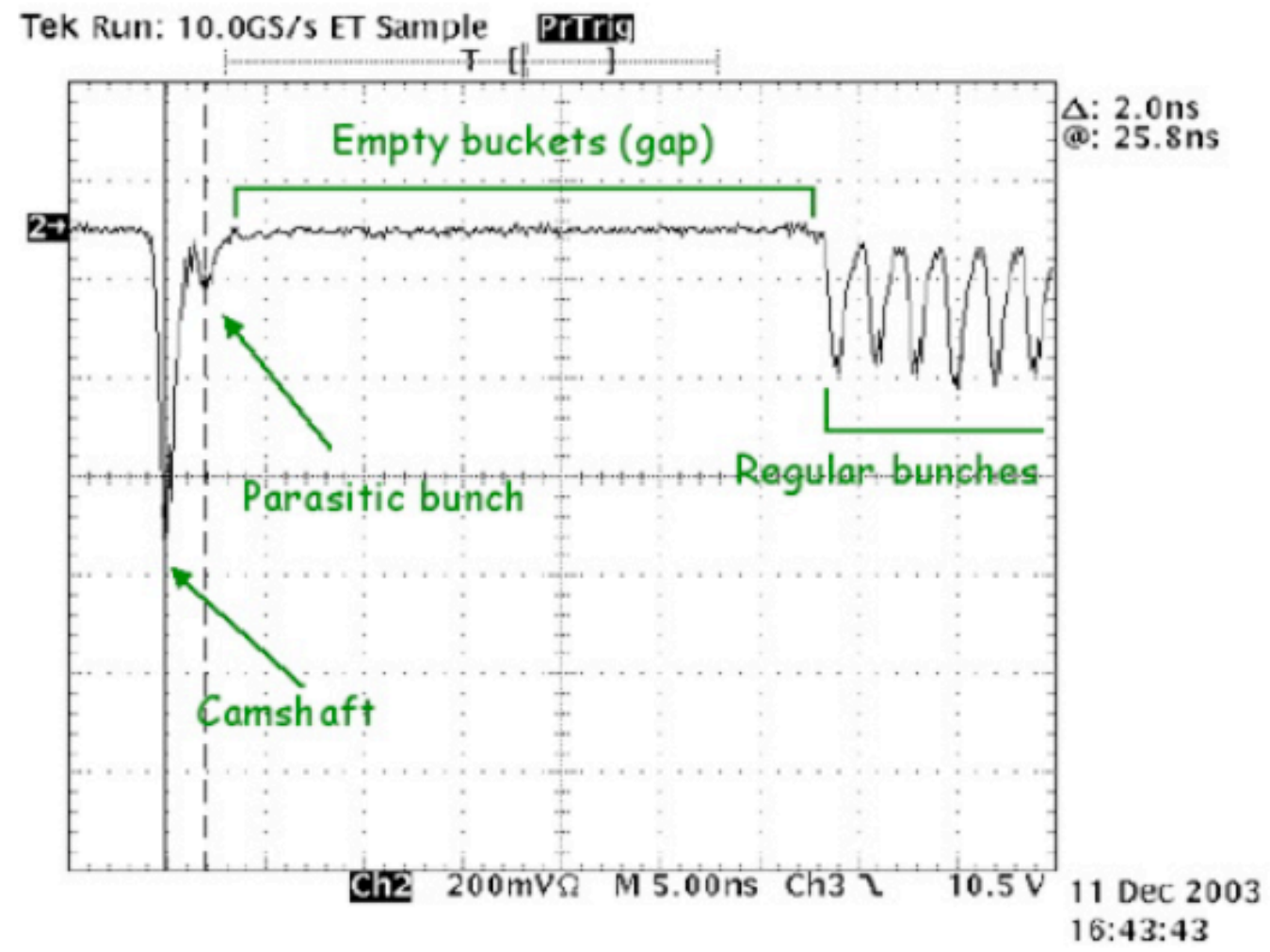

FIGURE 5. Image of the camshaft, trailing parasitic bunch and first 6 bunches of the ALS fill train. No averages.

After this additional attenuation is removed, Fig.5 gives an idea of the instrument time resolution. This image was taken with no averaging and shows the good signalto-noise characteristics of our instrument. 


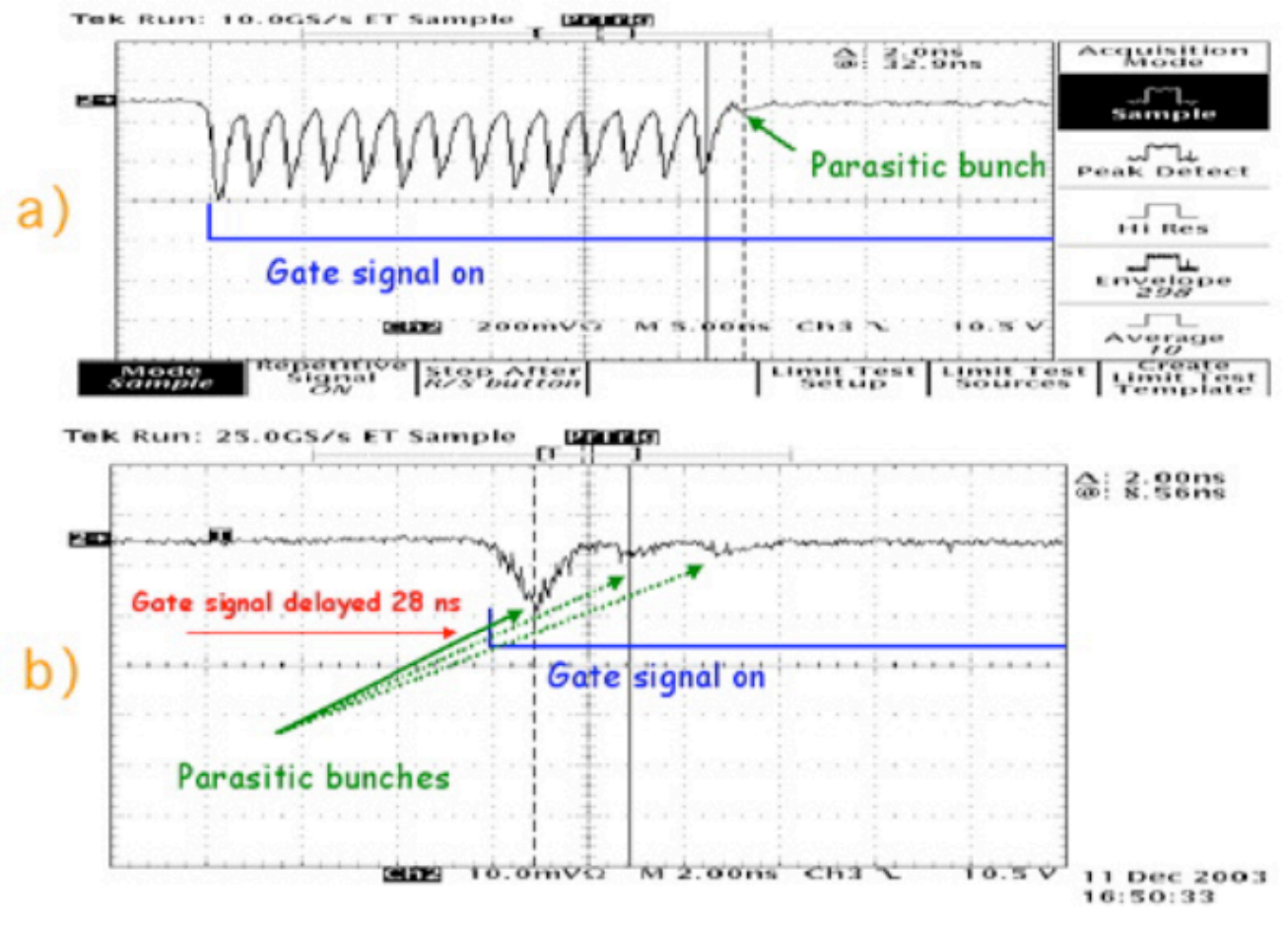

FIGURE 6. a) Beginning of the ALS gap and the last 14 filled RF buckets. b) Filled RF buckets are gated out by shifting the gate signal. Vertical scale is enlarged to show parasitic bunches.

In Fig.6 we show how it is possible to detect very low intensity bunches simply by gating out nearby large signals. The two parasitic bunches after the first one (which is also visible in Fig.6a) shown in Fig.6b have an estimated population of roughly $10^{7}$ electrons, about 100 times less than the regular bunches, showing that photocatode saturation is not an issue.

Figure 7 further demonstrate that transit time spread in the MCP-PMT has only limited effects on the measurement. Comparing the left side of the trace (with the gate off) to the rightmost side (gate on, no charges) shows the noise level of the instrument. Bunched particles are still easily detectable, even at low densities, since one knows where to look for them. Tests are being planned at the Tevatron to check the performance with unbunched beam. 


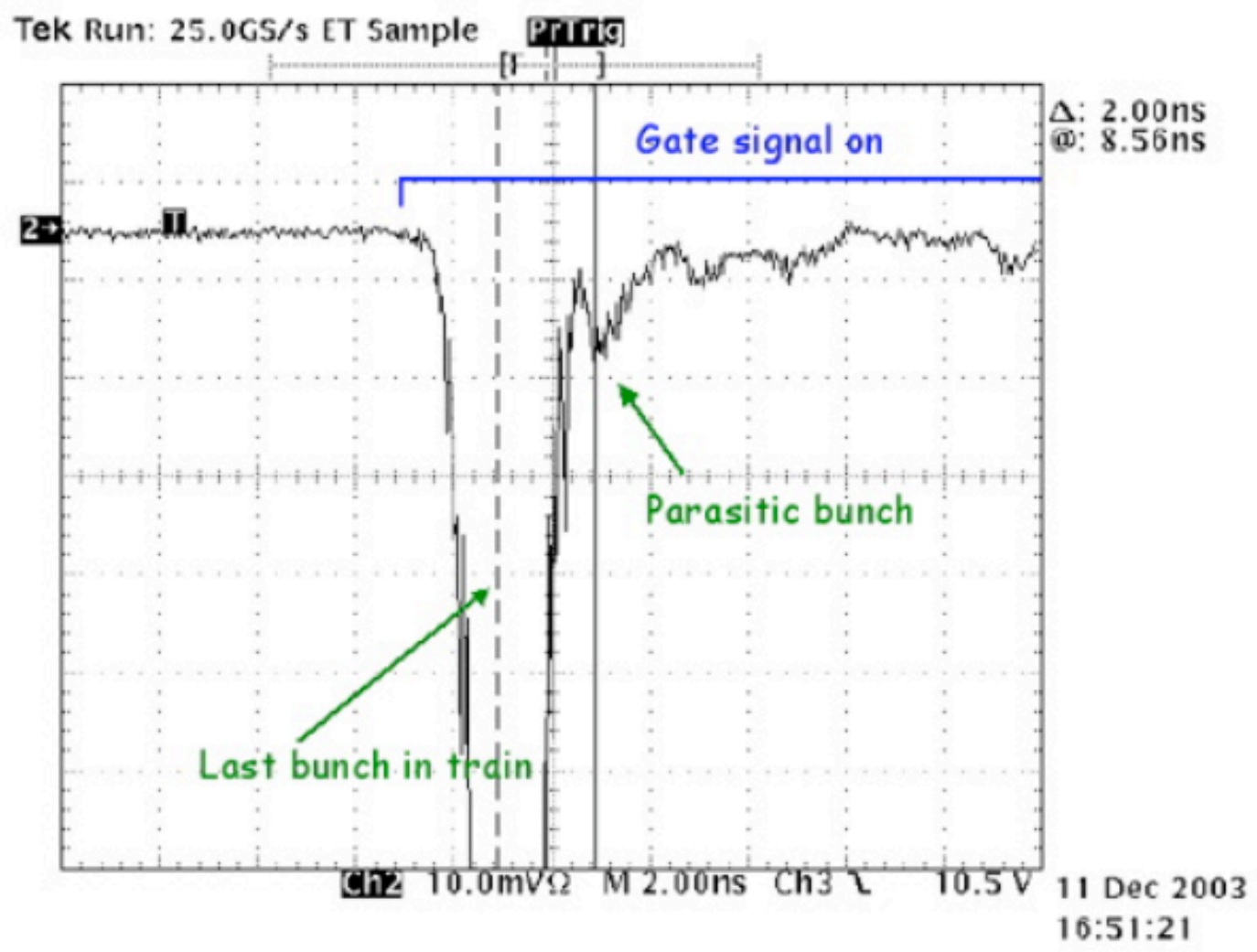

FIGURE 7. Close up view of the last bunch of the ALS train and the nearby parasitic bunch.

\section{IN CONCLUSIONS}

We have presented what we believe is a promising technique for monitoring the presence of low charge densities in the abort gap of storage rings. We use a gated microchannel plate photomultiplier for measuring the synchrotron radiation emitted and it is then possible to integrate the detected signal over the desired observation window to estimate the number of particles therein. The simplicity of the scheme proposed makes it suitable to be included in a machine interlock chain, where robustness and high MTBF are paramount.

Estimates of the signal levels obtainable in the LHC and experimental results at the ALS have also been shown.

\section{REFERENCES}

1. C. Fischer, High Sensitivity Measurement of the Longitudinal Distribution of the LHC Beams, LHCB-ES-0005, 2003.

2. http://www.hamamatsu.com.

3. M. Facchini and R. Jung, private communication. 